\begin{tabular}{|c|c|}
\hline Citation & $\begin{array}{l}\text { Vanhees I, Gunst J, (2013), } \\
\text { Enhanced immunoreceptor tyrosine-based activation motif signaling is } \\
\text { related to pathological bone resorption during critical illness. } \\
\text { Horm Metab Res. } 2013 \text { Nov; } 45(12): 862-9 \text {. Epub } 2013 \text { Aug. }\end{array}$ \\
\hline Archived version & $\begin{array}{l}\text { Author manuscript: the content is identical to the content of the } \\
\text { published paper, but without the final typesetting by the publisher }\end{array}$ \\
\hline Published version & http://dx.doi.org/10.1055/s-0033-1351290 \\
\hline Journal homepage & http://www.thieme.com/index.php?page=shop.product details\&flypage=flypag \\
\hline & e.tpl\&product id=957\&category id=90\&option=com_virtuemart\&ltemid=53 \\
\hline Author contact & $\begin{array}{l}\text { greet.vandenberghe@med.kuleuven.be } \\
\text { phone number + } 32(0) 16344021\end{array}$ \\
\hline IR & https://lirias.kuleuven.be/handle/123456789/420965 \\
\hline
\end{tabular}

(article begins on next page) 


\begin{tabular}{|c|c|}
\hline Citation & $\begin{array}{l}\text { Vanhees I, Gunst J, (2013), } \\
\text { Enhanced immunoreceptor tyrosine-based activation motif signaling is } \\
\text { related to pathological bone resorption during critical illness. } \\
\text { Horm Metab Res. } 2013 \mathrm{Nov} ; 45(12): 862-9 \text {. Epub } 2013 \text { Aug. }\end{array}$ \\
\hline Archived version & Final publisher's version / pdf \\
\hline Published version & http://dx.doi.org/10.1055/s-0033-1351290 \\
\hline Journal homepage & http://www.thieme.com/index.php?page=shop.product details\&flypage=flypag \\
\hline & e.tpl\&product id=957\&category id=90\&option=com virtuemart\&ltemid=53 \\
\hline Author contact & your email greet.vandenberghe@med.kuleuven.be \\
\hline & your phone number $+32(0) 16344021$ \\
\hline IR & https://lirias.kuleuven.be/handle/123456789/420965 \\
\hline
\end{tabular}

(article begins on next page) 


\title{
Enhanced Immunoreceptor Tyrosine-Based Activation Motif Signaling is Related to Pathological Bone Resorption during Critical Illness
}

\author{
Short title: Bone Loss during Critical Illness \\ Vanhees I. ${ }^{1}$, Gunst J. ${ }^{1}$, Janssens T. ${ }^{1}$, Wauters A. ${ }^{1}$, Van Herck E. ${ }^{2}$, \\ Van Cromphaut S. ${ }^{1}$, Van den Berghe G. ${ }^{1}$, Owen H.C. ${ }^{1}$ \\ ${ }^{1}$ Laboratory of Intensive Care Medicine, Department of Cellular and Molecular Medicine, \\ ${ }^{2}$ Laboratory of Experimental Medicine and Endocrinology, Department of Clinical and \\ Experimental Medicine, KU Leuven, Herestraat 49, B-3000 Leuven, Belgium \\ Email addresses: ineke.vanhees@med.kuleuven.be; jan.gunst@med.kuleuven.be; \\ thomas.janssens2@med.kuleuven.be; andy.wauters@med.kuleuven.be; \\ erik.vanherck@med.kuleuven.be; sophie.vancromphaut@med.kuleuven.be; \\ greet.vandenberghe@med.kuleuven.be; helen.owen@med.kuleuven.be
}

*Corresponding author and address for reprints: Dr. Helen C. Owen

KU Leuven

O\&N1, Herestraat 49 - Box 503

3000 Leuven

Belgium

Tel: +32/16/330 242

Fax: +32/16/330 932

helen.owen@med.kuleuven.be

Disclosures: The authors declare no potential conflicts of interest with respect to the authorship and/or publication of this article. 


\section{Abstract}

Prolonged critically ill patients present with distinct alterations in calcium and bone metabolism. Circulating bone formation markers are reduced and bone resorption markers are substantially elevated, indicating an uncoupling between osteoclast and osteoblast activity, possibly resulting in pronounced bone loss, impaired traumatic or surgical fracture healing and osteoporosis. In addition, we have previously shown that increased circulating osteoclast precursors in critically ill patients result in increased osteoclastogenesis in vitro, possibly through FcyRIII signaling. In the current study, we investigated the effects of sustained critical illness on bone metabolism at the tissue level in a standardized rabbit model of prolonged (7 days), burn injury-induced critical illness. This in vivo model showed a reduction in serum ionized calcium and osteocalcin levels, as is seen in humans. Trabecular area, bone mineral content and density were decreased in sick rabbits [by 43\% $(\mathrm{p}<0.01), 31 \%$ $(\mathrm{p}<0.01)$ and $29 \%(\mathrm{p}<0.05)$, respectively], as was the trabecular gene expression of osteoblast and angiogenesis markers, indicating decreased bone formation and impaired vascularization. There was no change in the expression of osteoclast differentiation markers from the canonical RANK/RANKL/OPG pathway, however, there was an increase in expression of markers from the non-canonical, immunoreceptor tyrosine-based activation motif (ITAM) signaling pathway, Fc $\gamma$ RIII and DAP12 (148\% and 59\%, respectively; $\mathrm{p}<0.01)$. The current study has shown a detrimental effect of prolonged critical illness on trabecular bone integrity, possibly explained by reduced osteoblast differentiation and angiogenesis, coupled with increased osteoclastogenesis signaling that may be mediated via the non-canonical immunoreceptor tyrosine-based activation motif signaling pathway.

\section{Keywords}

Intensive Care Unit; bone hyperresorption; osteoclast; osteoblast; ITAM 


\section{Introduction}

Advances in intensive care medicine over the past decades have made it possible for patients to survive a variety of previously lethal insults such as surgery, trauma, sepsis or extensive burn injury $[1,2]$. However, this has also created a growing population of patients who evolve towards a phase of prolonged critical illness, where they remain dependent upon vital organ function support for weeks or months. This chronic phase brings about a therapy-resistant catabolic state that is hallmarked by lean tissue wasting, skeletal muscle weakness and non-resolving multiple organ failure [1-3].

Normal bone turnover depends on a tight coupling between the function of mature osteoclasts, osteoblasts and vascularization, requiring a complex equilibrium of immune, endocrine, mechanical and nutritional factors [4]. The bone remodeling cycle is a closely regulated and carefully orchestrated sequence that involves coordinated communication between osteoblasts and osteoclasts [5]. Critically ill patients display vitamin D deficiency, hypocalcaemia, elevated levels of inflammatory cytokines such as tumor necrosis factor $\alpha(\mathrm{TNF}-\alpha)$, interleukin-1 (IL-1) and IL-6, and marked alterations in markers of bone metabolism [6-8]. More specifically, during prolonged critical illness levels of bone formation markers such as osteocalcin are low, and levels of bone resorption markers such as carboxyterminal cross-linked telopeptide of type I collagen are 6- to 15-fold increased. The degree of these alterations during critical illness is much higher than in classic metabolic diseases, such as postmenopausal osteoporosis, where only a 2-fold increase in bone resorption markers is observed [9]. Consequently, prolonged critically ill patients may be predisposed to inefficient traumatic or surgical fracture healing and to osteoporosis. Indeed, a recent retrospective case-cohort study revealed a significant increase in fracture risk among survivors of critical illness [10].

We recently showed that peripheral blood mononuclear cells (PBMCs) isolated from critically ill patients reveal an increased osteoclastogenic potential and differentiate into mature osteoclasts in vitro much more than cells from healthy subjects [11]. Moreover, the activity of the patients' mature osteoclasts appeared dependent on humoral factors present in the serum of critically ill patients. In particular, circulating humoral factors depending on signaling through ITAM receptors of the noncanonical pathway for osteoclastogenesis appeared to play a key role [12]. In inflammatory bone 
disorders such as rheumatoid arthritis, it has been shown that the expression of two immunomodulatory ITAM receptors, DNAX-activation protein 12 (DAP12) and Fc receptor common $\gamma$ chain III (Fc $\gamma$ RIII), is increased [13]. Blocking Fc $\gamma$ RIII in vitro decreased osteoclast formation in PBMCs from critically ill patients, suggesting that immunomodulatory factors present in patient serum may induce abberant osteoclast formation.

Although biomarkers in human patients suggest excessive bone loss during critical illness [8], as yet the in vivo effect of prolonged critical illness on bone integrity has not been documented. We assessed the effects of critical illness on trabecular bone in a well validated rabbit model of prolonged critical illness [14]. The current study showed a detrimental effect of critical illness on trabecular bone, possibly due to impaired osteoblast differentiation and activated osteoclastogenesis evoked by signaling through the ITAM signaling pathway.

\section{Materials and Methods}

\subsection{Animals and blood analysis}

The study protocol was approved by the KU Leuven Ethical Review Board for Animal Research (P108/2009). All animals were treated according to the Principles of Laboratory Animal Care (U.S. National Society for Medical Research) and the Guide for the Care and Use of Laboratory Animals (National Institutes of Health). The rabbit model of prolonged (7days) critical illness has been described in detail previously [14]. At day -1, adult, 3- to 4-month-old male New Zealand White rabbits $(n=40)$ were anesthetized and catheters were inserted in the right jugular vein and right carotid artery, allowing intravenous infusion of fluids and repetitive blood sampling, respectively. Fluid resuscitation consisting of Hartmann (Baxter, Lessines, Belgium), enriched with $5 \%$ glucose to prevent hypoglycemia, was started after the operation. At day 0 , animals were anesthetized again, and after having performed a paravertebral block with lidocaine (Xylocaine, AstraZeneca, Brussels, Belgium), a full thickness third-degree burn injury was inflicted on the flanks (15-20\% body surface area; painless by itself as cutaneous nerves for sensation of pain are destroyed). Hartmann-Glucose was changed to total parenteral nutrition [33.3\% Oliclinomel N7 (Baxter), 33.3\% Hartmann, and 
33.3\% Glucose 50\%] at day 1 . This model closely mimics the (neuro)endocrine, metabolic and clinical abnormalities of critically ill patients and has therefore been validated as representative of the human critically ill condition [4, 14]. Ionized calcium levels were measured immediately by blood gas analysis (ABL 725, Radiometer, Copenhagen, Denmark) peroperatively at day -1, before burn injury at day 0 and from day 1 every morning. At the same time, a daily $4 \mathrm{ml}$ blood sample was collected and plasma was stored at $-80^{\circ} \mathrm{C}$ until further analysis. Animals surviving until day $7 \quad(n=30)$ were anesthetized and were sacrificed by cardiectomy. Animals entering a preterminal state before day 7 $(n=10)$, as evidenced clinically and by blood gas analysis, were anesthetized and sacrificed at that time. The left and right tibiae were dissected, dipped in polyvinyl alcohol and snap-frozen in liquid nitrogen, and stored at $-80^{\circ} \mathrm{C}$ until further analysis. For comparison, healthy rabbits $(n=15)$ were sacrificed and tissue samples were collected as described above. These rabbits did not receive a burn injury, were not catheterized and had free access to standard chow. In every experiment, all critically ill rabbits (survivors and non-survivors) are compared to the healthy controls, unless otherwise indicated. Plasma osteocalcin was measured by an in-house rabbit osteocalcin radioimmunoassay as previously described $[8,15]$.

\subsection{Bone quantification}

Peripheral quantitative computed tomography (pQCT; Stratec XCT Research densitometer, Norland Medical Systems, Fort Atkinson, WI, USA) was used to assess trabecular bone mineral content (BMC) and density (BMD) and the geometry of the proximal right tibia from critically ill rabbits ( $n=27$ survivors and 7 non-survivors) and healthy controls $(n=15)$ ex vivo. Using a voxel size of 0.070 $\mathrm{mm}$, slices of $0.2 \mathrm{~mm}$ thickness were obtained. Three metaphyseal scans were taken $2.4 \mathrm{~mm}$ from the proximal end of the tibia to measure trabecular volumetric density. An inner threshold was set to $30 \%$ of the total cross-sectional area to define the trabecular bone region. Following pQCT, the proximal tibiae of these rabbits were decalcified in $10 \%$ EDTA at $4^{\circ} \mathrm{C}$ in order to enable sectioning of tissues [16]. After 6 weeks, the tibiae were processed to paraffin and sections of $5 \mu \mathrm{m}$ thickness were cut. 


\subsection{Histological and immunohistochemical analysis of rabbit trabecular bone}

Sections from the right proximal tibia from 15 healthy controls and prolonged critically ill rabbits were stained for H\&E, and the amount of trabecular bone quantified by Image $J^{\circledR}$ software (National Institutes of Health) [17]. The amount of demineralized, mature bone vs. unmineralized osteoid or immature bone present was histologically analyzed through a Masson's Trichrome stain as previously described [18]. The amount of immature bone (red color) vs. mature bone (blue color) was visualized using fluorescent microscopy and quantified using an in-house software tool. Osteoclast activity was quantified visually with tartrate-resistant acid phosphatase (TRAP), as previously described [11]. The osteoclastogenic marker FcyRIII was detected by immunohistochemistry with goat-anti-rabbit polyclonal antibody (1:200; Antibodies-online, Aachen, Germany), followed by probing with donkey anti-goat IgG (1:500; Jackson Immunoresearch, West Grove, PA, USA). Fc $\gamma$ RIII-positive cells were visualized and quantified manually.

\subsection{RNA isolation and quantitative PCR}

Gene expression analysis was performed on the trabecular bone of the right tibia of critically ill rabbits ( $\mathrm{n}=3$ survivors and 2 non-survivors) and healthy controls ( $\mathrm{n}=5$ ). Total RNA was isolated using the RNeasy mini RNA isolation kit (QIAGEN) according to manufacturer’s instructions and quantified by Nanodrop ND-1000 Spectrophotometer (Nanodrop Technologies, Wilmington, DE, USA). In total, 1 $\mu$ g RNA was reverse-transcribed using SuperScript III Reverse Transcriptase (Invitrogen, Merelbeke, Belgium). All samples were reverse transcribed simultaneously. mRNA levels were quantified in realtime using the StepOnePlus ${ }^{\mathrm{TM}}$ Real-Time PCR System (Applied Biosystems, Chershire, UK). SYBR ${ }^{\circledR}$ Green qPCR primers were designed using the software program Primer3 (Whitehead Institute for Biomedical Research, Cambridge, MA, USA) to span an intron so that only RNA-specific amplification was possible (Table S1). The gene expression of 3 osteogenic markers, namely runtrelated transcription factor 2 (RUNX2), osterix (OSX) and collagen type I alpha 1 (COL1A1) was measured. For osteoclastogenesis, the gene expression of markers of 2 different signaling pathways was measured: receptor activator of nuclear factor kappa-B ligand (RANKL), as well as its receptor RANK and decoy receptor osteoprotegerin (OPG) as markers of the canonical RANK/RANKL/OPG 
pathway, and DAP12 and Fc $\gamma$ RIII, both involved in the alternative ITAM signaling pathway. Vascular endothelial growth factor receptor 1 (VEGFR1) and VEGFR2 represent two markers of angiogenesis. Unknown samples were run in triplicate. Relative fold changes in expression were calculated using the $\Delta \mathrm{Ct}$ method [19]. Gene expression of housekeeping gene hypoxanthine-guanine phosphoribosyltransferase (HPRT) was used as an internal control.

\subsection{Statistical analysis}

Data are presented as mean \pm standard error of the mean (SEM), median and interquartile ranges box plots, with $10^{\text {th }}$ and $90^{\text {th }}$ percentiles or as numbers and percentages. Student's $t$ test was used for the comparison of normally distributed data and the Mann-Whitney U test for data that were not normally distributed. All statistical analyses were calculated using Statview 5.0.1. software (SAS Institute Inc., Cary, NC, USA). Statistical significance is indicated on all graphs as follows: *: $\mathrm{p}<0.05, * *$ : $\mathrm{p}<0.01$, $* * *: \mathrm{p}<0.001$.

\section{Results}

\subsection{Serum ionized calcium and plasma osteocalcin levels are decreased in critically ill rabbits}

In order to confirm that the in vivo rabbit model of prolonged critical illness utilized in the current study was a suitable representation of bone metabolism in prolonged critically ill humans, we measured serum ionized calcium and plasma osteocalcin levels. As in critically ill patients, serum ionized calcium was significantly reduced in critically ill rabbits (Fig. 1A). Infliction of the critical illness evoked an immediate decrease in ionized calcium on day $1(3 \%$; $<<0.01)$, which remained lower than in healthy rabbits for the 7-day period of illness. In addition, plasma osteocalcin levels were significantly lowered in critically ill rabbits by day 4 of illness [by 62\% ( $\mathrm{p}<0.001)$ ] (Fig. 1B), similar to what is seen in critically ill patients. The survivor status of the critically ill rabbits did not significantly affect the results (data not shown). 


\subsection{Trabecular bone is reduced in critically ill rabbits}

In order to examine the direct effect of critical illness on the skeleton, BMC and BMD were analyzed ex vivo in the proximal tibia through pQCT. Importantly, trabecular BMC and BMD were significantly lower in critically ill rabbits than in healthy controls [31\% $(\mathrm{p}<0.01)$ and 29\% $(\mathrm{p}<0.05)$, respectively]

(Fig. 1C, D). The reduction in trabecular bone was confirmed histologically with a significant $43 \%$ reduction $(\mathrm{p}<0.001)$ in average trabecular area in critically ill rabbits as compared to healthy controls (Fig. 1E, F). Survivor status of the critically ill rabbits did not significantly affect these results (data not shown).

3.3 Histological increases in osteoclast activity are undetectable in trabecular bone at day 7 in critically ill rabbits

Masson's Trichrome staining did not reveal a difference in the amount of mature and immature bone present in critically ill rabbits as compared to healthy controls (Fig. 2A). In addition, TRAP staining and Fc $\gamma$ RIII immunohistochemistry failed to show any significant difference in the number of active osteoclasts in the trabecular bone of the proximal tibiae of critically ill rabbits and healthy controls (Fig. 2B, C). We cannot exclude that increases in osteoclast activity may have occurred at earlier time points.

3.4 Critically ill rabbits present with a decrease in gene expression of osteogenic and angiogenic markers and an increase in markers of the osteoclastogenic ITAM signaling pathway

To investigate osteoblast differentiation further, the gene expression of RUNX2, OSX and COL1A1 was measured in the trabecular bone of prolonged critically ill and healthy control rabbits. The expression of these 3 markers was significantly decreased in the critically ill rabbits as compared to healthy controls [by 55\% ( $<<0.05), 61 \%(\mathrm{p}<0.01)$ and $74 \%(\mathrm{p}<0.01)$, respectively] (Fig. 3A-C). VEGFR1 and -2 gene expression was measured as a marker of angiogenesis during critical illness. VEGFR2 expression was significantly decreased in critically ill rabbits as compared to healthy controls (59\%; $\mathrm{p}<0.01)$, while the expression of VEGFR1 displayed a trend towards a reduction, although did not reach significance (58\%; p=0.08) (Fig. 3D, E). Osteoclastogenesis was assessed 
through the gene expression of markers of 2 different signaling pathways, from which the canonical RANK/RANKL/OPG pathway is the most common osteoclastogenic pathway. A significant reduction in the gene expression of RANKL in the critically ill rabbits as compared to healthy controls was observed (62\%; $\mathrm{p}<0.01)$, along with a trend for a decreased expression of OPG (63\%; $\mathrm{p}=0.07)$, resulting in an unchanged OPG/RANKL ratio, suggesting this pathway may be unaltered during critical illness (Fig. 4A-C). An alternative osteoclastogenic ITAM signaling pathway, including FcyRIII and DAP12 as 2 important ITAM receptors, is known to be involved in diseases of extreme bone resorption [12, 20], and interestingly, gene expression of Fc $\gamma$ RIII and DAP12 was increased significantly (148\% and 59\%, respectively; p<0.01) (Fig. 4D, E). Survivor status of the critically ill rabbits did not significantly affect these results (data not shown).

\section{Discussion}

During prolonged critical illness levels of bone formation markers are low, and levels of bone resorption markers are 6- to 15-fold increased. Critical illness, whether or not evoked by burn injuries, is characterized by a marked hypermetabolic response, hypercatabolism, and a severe loss of lean body mass [21]. A mouse model with burn injury displayed a 59\% reduction in trabecular BMC compared to controls after 10 days [22]. In addition, a number of studies have shown that children with burn injuries exceeding $40 \%$ total body surface area are subject to abnormalities in bone and calcium metabolism including acute and chronic bone loss, early post-burn hypocalcaemia and hypoparathyroidism [23]. The bone loss is manifested by an approximate $2 \%$ loss of total body BMC 2 months after the burn injury, increasing to 3\% by 6 months, with lumbar spine BMC falling by $8 \%$ after 2 months. However, while the catabolic state eventually resolves, BMD remains low in these children for years following the burn injury [24], resulting in an increased risk of fracture. These findings suggest a direct association between the extreme catabolic state experienced during critical illness or burn injury and subsequent skeletal morbidity. This is confirmed by a recent retrospective longitudinal case-cohort study by Orford and colleagues revealing an increased risk of fragility fractures over an 8-year period in elderly female survivors of critical illness [10]. We recently reported 
in an in vitro study using human cells that impaired angiogenesis and osteoclastic abnormalities most likely mediated by immunomodulatory factors through the non-canonical ITAM signaling pathway, may contribute to critical illness related bone loss in the intensive care unit [11]. In the current study, we sought to confirm and further investigate these findings in an in vivo rabbit model of critical illness.

As previously reported, the rabbit model of prolonged critical illness mirrors many of the endocrine and metabolic changes observed in critically ill patients [14]. In the current study, critically ill rabbits displayed low levels of serum ionized calcium throughout the 7-day period of illness. During critical illness in patients, abnormalities in ionized calcium levels are common, with $90 \%$ of patients displaying mild hypocalcaemia, although hypercalcaemia has also been reported in the prolonged phase of illness, particularly when renal failure is present [25]. The observed reduction in plasma osteocalcin in critically ill rabbits after 4 days is also in line with findings in critically ill patients [8], suggesting that bone formation may be reduced during critical illness. This was confirmed by the current study, in which the trabecular bone in tibiae of critically ill rabbits revealed a $31 \%$ and $29 \%$ reduction of mineral content and density, respectively, and a $43 \%$ decrease in area as compared with healthy control rabbits. It has been shown that corticosteroid treatment can produce osteoporosis with $25 \mathrm{mg}$ of prednisone on alternate days leading to an average reduction of 3.5\% of trabecular bone over 1 year [26], and similarly, in a rabbit model of osteoporosis evoked by methylprednisolone, trabecular BMD was reduced by $36 \%$ after 10 weeks of treatment [27]. Therefore, our observation of reduced trabecular bone after only 7 days of critical illness is remarkable. It is possible that such a large decrease in trabecular bone could be due, in part, to immobilization, which induces skeletal unloading leading to an imbalance between bone resorption and bone formation, resulting in trabecular bone loss [28]. However, in the current study, rabbits have adequate space for limited movement, and critically ill rabbits are able to use their front- and hind-limbs to support their weight. Critically ill patients are also immobilized for long periods of time, however a recent study showed only a 2-fold increase in the bone resorption marker beta-carboxy-terminal crosslinking telopeptide I ( $\beta$-CTX-I) in patients that were immobilized by stroke for 6 months, suggesting that immobilization alone cannot explain the remarkable 6-fold increase in bone resorption markers that is observed in prolonged critical illness 
[29]. Surprisingly, the reduction in trabecular BMC and BMD was not associated with an increase in TRAP- or FcyRIII-positive multinuclear osteoclasts. It is possible that increases in osteoclast activity may have occurred at earlier time points, resulting in the extreme bone loss observed.

In other metabolic bone diseases such as Paget's disease of bone and osteoporosis, reduction in trabecular BMD is thought to be caused by an 'uncoupling' between bone forming osteoblasts and bone resorbing osteoclasts. In the current study, there was a significant decrease in the expression of early (RUNX2 and OSX) and late (COL1A1) markers of osteoblast differentiation. This finding, coupled with a reduction in the expression of angiogenesis marker VEGFR2 suggests a potential rationale for the reduction in trabecular bone observed, and confirms our previous in vivo study where a reduction in bone formation and vascularization was observed in patient serum-treated human periosteal derived cells seeded onto calcium phosphate scaffolds and implanted into mice for 8 weeks [11]. Recently, it has been shown that healthy human PBMCs can be subdivided into two subsets, FcrRIII-positive and -negative monocytes, from which the latter is known to differentiate into mature osteoclasts in vitro only after stimulation with RANKL and macrophage colony-stimulating factor MCSF [30]. However, it has been shown that there is an upregulation of circulating FcyRIII-positive monocytes in inflammatory bone diseases. These cells represented the major reservoir of osteoclast precursors in inflammatory diseases and the level of FcyRIII expression correlated with the bone resorption activity [12]. In critically ill patients, we have previously shown that osteoclast precursors differentiate into mature active osteoclasts even in the absence of RANKL and MCSF, suggesting that the majority of circulating monocytes in critically ill patients are FcyRIII-positive. This was confirmed when, by blocking the FcyRIII receptor in critically ill PBMCs in vitro, aberrant osteoclast formation was ameliorated [11]. These data imply that during critical illness, osteoclastogenesis is potentially due to circulating humoral factors and/or IgG antibodies and driven through the non-canonical ITAM signaling pathway rather than the common RANK/RANKL/OPG pathway [11]. Interestingly, in some inflammatory diseases of bone hyperresorption such as psoriatic arthritis, rheumatoid arthritis and osteoporosis, alternative signaling via the ITAM receptors Fc $\gamma$ RIII and DAP12 has been proposed as the pathway through which osteoclast formation and activity is stimulated [13, 31, 32]. Although in the current study, OPG/RANKL ratio was unchanged and no significant differences in TRAP staining 
or FcyRIII immunohistochemistry could be detected after 7 days of illness, there was a significant increase in the gene expression of FcyRIII and DAP12 (148\% and 59\%, respectively). This supports the hypothesis that signaling through the canonical pathway was unaffected by critical illness, however, the observed critical illness-induced reduction in trabecular bone may be mediated via noncanonical ITAM signaling pathway-driven osteoclastogenesis.

\section{Conclusions}

The present study has investigated bone metabolism at tissue level during critical illness in a validated animal model for prolonged critical illness. The critical illness-related reduction in trabecular bone may result from osteoclastic bone hyperresorption mediated through the non-canonical ITAM signaling pathway combined with a decrease in osteoblast formation and angiogenesis (Figure S1). These findings may help to define novel therapeutic targets to prevent critical illness-induced bone loss.

\section{References}

[1] Van den Berghe G, de Zegher F, Bouillon R. Clinical review 95: Acute and prolonged critical illness as different neuroendocrine paradigms. J Clin Endocrinol Metab 1998; 83: 1827-1834

[2] Nelson JE, Cox CE, Hope AA, Carson SS. Chronic critical illness. Am J Respir Crit Care Med 2010; 182: 446-454

[3] Hermans G, Vanhorebeek I, Derde S, Van den Berghe G. Metabolic aspects of critical illness polyneuromyopathy. Crit Care Med 2009; 37: S391-397

[4] Van den Berghe G. Endocrine evaluation of patients with critical illness. Endocrinol Metab Clin North Am 2003; 32: 385-410

[5] Parfitt AM. The bone remodeling compartment: a circulatory function for bone lining cells. J Bone Miner Res 2001; 16: 1583-1585

[6] Nierman DM, Mechanick JI. Biochemical response to treatment of bone hyperresorption in chronically critically ill patients. Chest 2000; 118: 761-766 
[7] Smith LM, Cuthbertson B, Harvie J, Webster N, Robins S, Ralston SH. Increased bone resorption in the critically ill: association with sepsis and increased nitric oxide production. Crit Care Med 2002; 30: 837-840

[8] Van den Berghe G, Van Roosbroeck D, Vanhove P, Wouters PJ, De Pourcq L, Bouillon R. Bone turnover in prolonged critical illness: effect of vitamin D. J Clin Endocrinol Metab 2003; 88: $4623-4632$

[9] Fink E, Cormier C, Steinmetz P, Kindermans C, Le Bouc Y, Souberbielle JC. Differences in the capacity of several biochemical bone markers to assess high bone turnover in early menopause and response to alendronate therapy. Osteoporos Int 2000; 11: 295-303

[10] Orford NR, Saunders K, Merriman E, Henry M, Pasco J, Stow P, Kotowicz M. Skeletal morbidity among survivors of critical illness. Crit Care Med 2011; 39: 1295-1300

[11] Owen HC, Vanhees I, Solie L, Roberts SJ, Wauters A, Luyten FP, Van Cromphaut S, Van den Berghe G. Critical illness-related bone loss is associated with osteoclastic and angiogenic abnormalities. J Bone Miner Res 2012; 27: 1541-1552

[12] Chiu YG, Shao T, Feng C, Mensah KA, Thullen M, Schwarz EM, Ritchlin CT. CD16 (FcRgammaIII) as a potential marker of osteoclast precursors in psoriatic arthritis. Arthritis Res Ther 2010; 12: R14

[13] Crotti TN, Dharmapatni AA, Alias E, Zannettino AC, Smith MD, Haynes DR. The immunoreceptor tyrosine-based activation motif (ITAM) -related factors are increased in synovial tissue and vasculature of rheumatoid arthritic joints. Arthritis Res Ther 2012; 14: R245

[14] Weekers F, Giulietti AP, Michalaki M, Coopmans W, Van Herck E, Mathieu C, Van den Berghe G. Metabolic, endocrine, and immune effects of stress hyperglycemia in a rabbit model of prolonged critical illness. Endocrinology 2003; 144: 5329-5338

[15] Bouillon R, Vanderschueren D, Van Herck E, Nielsen HK, Bex M, Heyns W, Van Baelen H. Homologous radioimmunoassay of human osteocalcin. Clin Chem 1992; 38: 2055-2060

[16] Sanjai K, Kumarswamy J, Patil A, Papaiah L, Jayaram S, Krishnan L. Evaluation and comparison of decalcification agents on the human teeth. J Oral Maxillofac Pathol 2012; 16: 222-227 
[17] Roberts SJ, Geris L, Kerckhofs G, Desmet E, Schrooten J, Luyten FP The combined bone forming capacity of human periosteal derived cells and calcium phosphates. Biomaterials 2011; 32:4393-4405

[18] Jaquiery C, Schaeren S, Farhadi J, Mainil-Varlet P, Kunz C, Zeilhofer HF, Heberer M, Martin I. In vitro osteogenic differentiation and in vivo bone-forming capacity of human isogenic jaw periosteal cells and bone marrow stromal cells. Ann Surg 2005; 242: 859-67, discussion 867-868

[19] Livak KJ, Schmittgen TD. Analysis of relative gene expression data using real-time quantitative PCR and the 2(-Delta Delta C(T)) Method. Methods 2001; 25: 402-408

[20] Mocsai A, Humphrey MB, Van Ziffle JA, Hu Y, Burghardt A, Spusta SC, Majumdar S, Lanier LL, Lowell CA, Nakamura MC. The immunomodulatory adapter proteins DAP12 and Fc receptor gamma-chain (FcRgamma) regulate development of functional osteoclasts through the Syk tyrosine kinase. Proc Natl Acad Sci U S A 2004; 101: 6158-6163

[21] Dominioni L, Trocki O, Fang CH, Mochizuki H, Ray MB, Ogle CK, Alexander JW. Enteral feeding in burn hypermetabolism: nutritional and metabolic effects of different levels of calorie and protein intake. JPEN J Parenter Enteral Nutr 1985; 9: 269-279

[22] Miller SC, Bowman BM, Siska CC, Shelby J. Effects of thermal injury on skeletal metabolism in two strains of mice. Calcif Tissue Int 2002; 71: 429-436

[23] Klein GL, Herndon DN, Langman CB, Rutan TC, Young WE, Pembleton G, Nusynowitz M, Barnett JL, Broemeling LD, Sailer DE, et al. Long-term reduction in bone mass after severe burn injury in children. J Pediatr 1995; 126: 252-256

[24] Klein GL, Herndon DN, Goodman WG, Langman CB, Phillips WA, Dickson IR, Eastell R, Naylor KE, Maloney NA, Desai M, et al. Histomorphometric and biochemical characterization of bone following acute severe burns in children. Bone 1995; 17: 455-460

[25] Egi M, Kim I, Nichol A, Stachowski E, French CJ, Hart GK, Hegarty C, Bailey M, Bellomo R. Ionized calcium concentration and outcome in critical illness. Crit Care Med 2011; 39: 314-321

[26] Ruegsegger P, Medici TC, Anliker M. Corticosteroid-induced bone loss. A longitudinal study of alternate day therapy in patients with bronchial asthma using quantitative computed tomography. Eur J Clin Pharmacol 1983; 25: 615-620 
[27] Baofeng L, Zhi Y, Bei C, Guolin M, Qingshui Y, Jian L. Characterization of a rabbit osteoporosis model induced by ovariectomy and glucocorticoid. Acta Orthop 2011; 81: 396-401

[28] Hott M, Deloffre P, Tsouderos Y, Marie PJ. S12911-2 reduces bone loss induced by shortterm immobilization in rats. Bone 2003; 33: 115-123

[29] Gaudio A, Pennisi P, Bratengeier C, Torrisi V, Lindner B, Mangiafico RA, Pulvirenti I, Hawa G, Tringali G, Fiore CE. Increased sclerostin serum levels associated with bone formation and resorption markers in patients with immobilization-induced bone loss. J Clin Endocrinol Metab 2010; 95: $2248-2253$

[30] Zhao R. Immune regulation of osteoclast function in postmenopausal osteoporosis: a critical interdisciplinary perspective. Int J Med Sci 2012; 9: 825-832

[31] Chiu YH, Mensah KA, Schwarz EM, Ju Y, Takahata M, Feng C, McMahon LA, Hicks DG, Panepento B, Keng PC, Ritchlin CT. Regulation of human osteoclast development by dendritic cellspecific transmembrane protein (DC-STAMP). J Bone Miner Res 2012; 27: 79-92

[32] Nose M, Yamazaki H, Hagino H, Morio Y, Hayashi S, Teshima R. Comparison of osteoclast precursors in peripheral blood mononuclear cells from rheumatoid arthritis and osteoporosis patients. J Bone Miner Metab 2009; 27: 57-65

\section{Figure Legends}

Figure 1. Blood analysis and trabecular bone quantification of the proximal tibiae in critically ill rabbits. (A) Serum ionized calcium levels were reduced from day 1 (by 3\%) in critically ill rabbits (grey dots; mean \pm SEM; $n=40$ ) and remained lower than in healthy rabbits (white dots; $n=15$ ) for the 7-day period of illness. (B) Plasma osteocalcin levels were significantly lower in critically ill rabbits (grey box plots; $n=40$ ) as compared to healthy controls (white box plots; $n=15$ ) by day 4 of illness (62\%). Quantification by pQCT revealed that trabecular BMC (C) and BMD (D) were significantly lower in critically ill rabbits (grey box plots; $n=34$ ) than in healthy controls (white box plots; $n=15$ ) (31\% and 29\%, respectively). (E) H\&E staining of the proximal tibiae of critically ill rabbits and healthy controls ( $\mathrm{n}=10$ for both groups). (F) Quantification of the H\&E staining using Image ${ }^{\circledR}$ 
revealed a significant reduction (43\%) in trabecular area in critically ill rabbits as compared to healthy controls. Data in panels B-F are represented as box plots, with medians, interquartile ranges and $10^{\text {th }}$ and $90^{\text {th }}$ percentiles; * $\mathrm{p}<0.05 ; * * \mathrm{p}<0.01 ; * * * \mathrm{p}<0.001$.

Figure 2. Histological and immunohistochemical analysis of critically ill rabbit trabecular bone. (A) Masson's Trichrome stain did not reveal a difference in the amount of unmineralized osteoid or immature bone (red) and demineralized, mature bone (blue) present in critically ill rabbits compared to

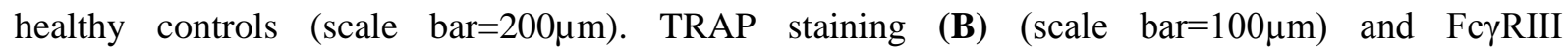
immunohistochemistry (C) (scale bar=50 $\mu$ m) of the proximal tibia did not show a significant difference in the number of active osteoclasts (black arrows) in the trabecular bone of critically ill rabbits and healthy controls. $\mathrm{n}=10$ for both groups.

Figure 3. Expression of osteogenic and angiogenic markers in critically ill rabbit trabecular bone. The expression of 3 osteogenic markers, RUNX2 (A), OSX (B) and COL1A1 (C) were significantly reduced in critically ill rabbits as compared to healthy controls (by 55\%, $61 \%$ and $74 \%$, respectively). Angiogenesis markers VEGFR1 (D) and VEGFR2 (E) also revealed a decrease in critically ill rabbits as compared to healthy controls ( $58 \%$ and $59 \%$, respectively). $n=5$ for both groups; ${ }^{*} \mathrm{p}<0.05 ; * * \mathrm{p}<0.01$

Figure 4. Expression of osteoclast markers in critically ill rabbit trabecular bone. (A) RANKL expression was significantly reduced in critically ill rabbits as compared to healthy controls (by 62\%); there was a trend $(\mathrm{p}=0.07)$ for decreased expression in OPG (B), resulting in no change in the OPG/RANKL ratio (C). The gene expression FcyRIII (D) and DAP12 (E), were significantly increased (148\% and 59\%, respectively) in critically ill rabbits as compared to healthy controls. $n=5$ for both groups; * $\mathrm{p}<0.05 ; * * \mathrm{p}<0.01$.

Figure S1. Proposed mechanism of bone loss during prolonged critical illness. The reduction in trabecular bone during critical illness is possibly a direct result of bone hyperresorption through aberrant osteoclast formation that may be mediated via the non-canonical ITAM signaling pathway, combined with a decrease in osteoblast formation and angiogenesis. (Dotted lines represent unchanged signaling; full lines represent increased reactions).

Table S1. Sequences of qPCR primers 


\section{Acknowledgements}

We thank Annelies Aertgeerts and Eric-Jan Ververs for their excellent technical assistance with regards to the rabbit experiment. We are grateful to Tyco Healthcare (Mechelen, Belgium) for instrumental and nutritional supplies This work was supported by the Fund for Scientific Research (FWO), Flanders, Belgium (SVC) and by the Research Council of the University of Leuven (GOA2007/14 to GVdB; F+/10/003 to HO). JG received a FWO Research Assistant Fellowship. GVdB, via the KU Leuven, received long-term structural research financing through the Methusalem program (METH08) funded by the Flemish government and has been awarded a "European Research Council Advanced grant” (ERC-A-dvG-2012-32/670) from the 'Ideas program' of the European Union $7^{\text {th }}$ framework.

Authors’ roles: Study design: IV, JG, TJ, SV, GVdB and HO. Study conduct: IV, JG and HO. Data collection: IV, JG, AW, EVH and HO. Data analysis: IV, JG, TJ, HO. Data interpretation: IV, SVC, GVdB and HO. Drafting manuscript: IV and HO. Revising manuscript content: IV, JG, TJ, AW, EVH, SVC, GVdB and HO. Approving final version of manuscript: IV, JG, TJ, AW, EVH, SVC, GVdB and HO. IV takes responsibility for the integrity of the data analysis. 
A

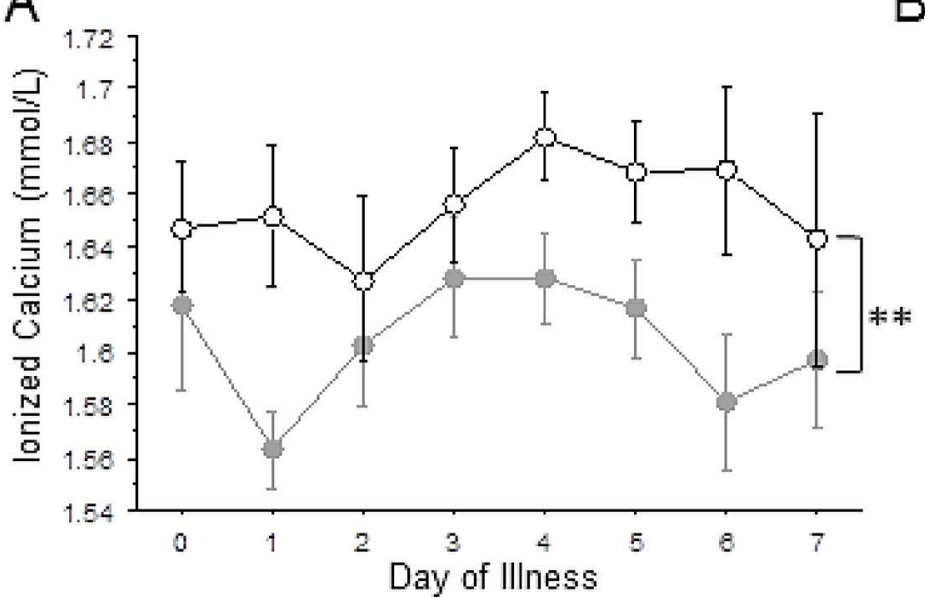

C

$$
\text { E }
$$

D

F
B 700
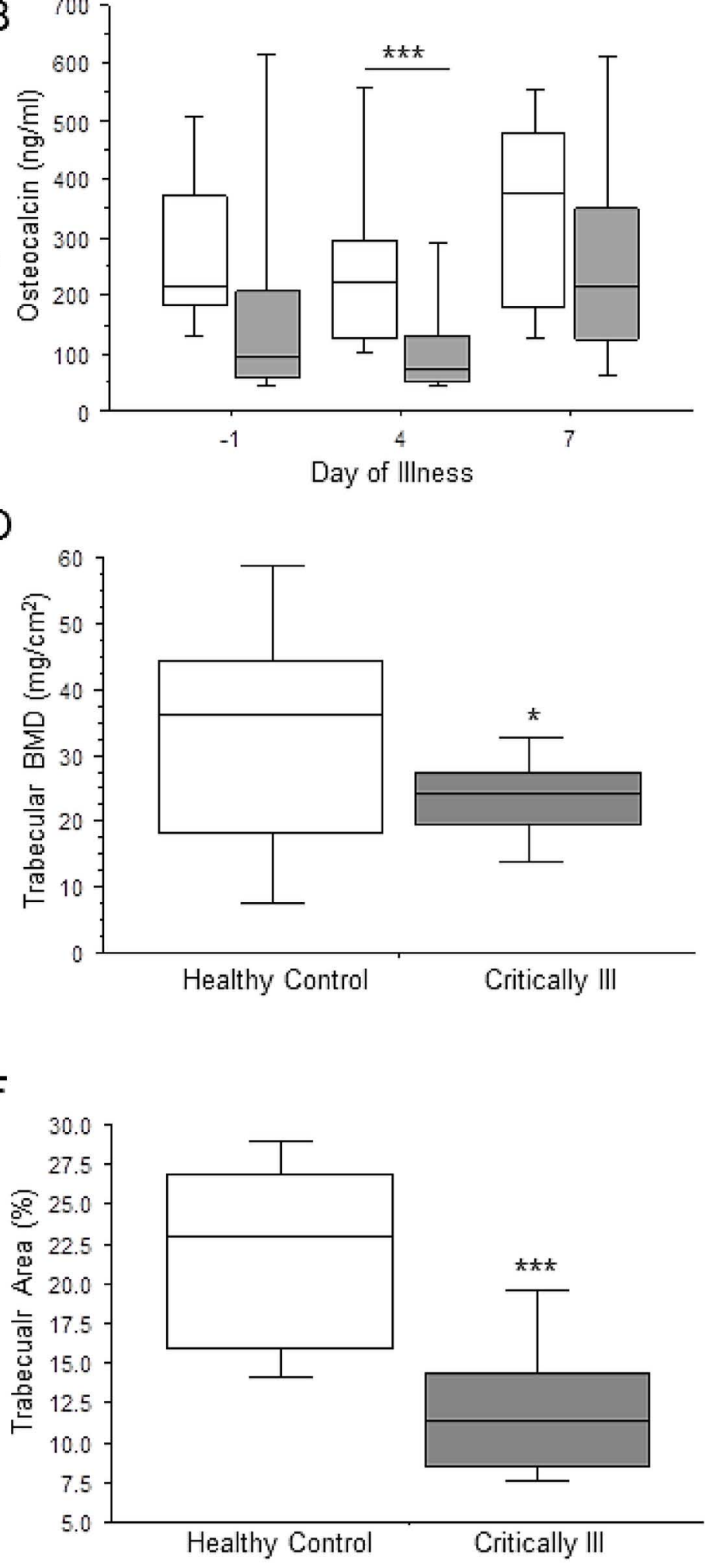


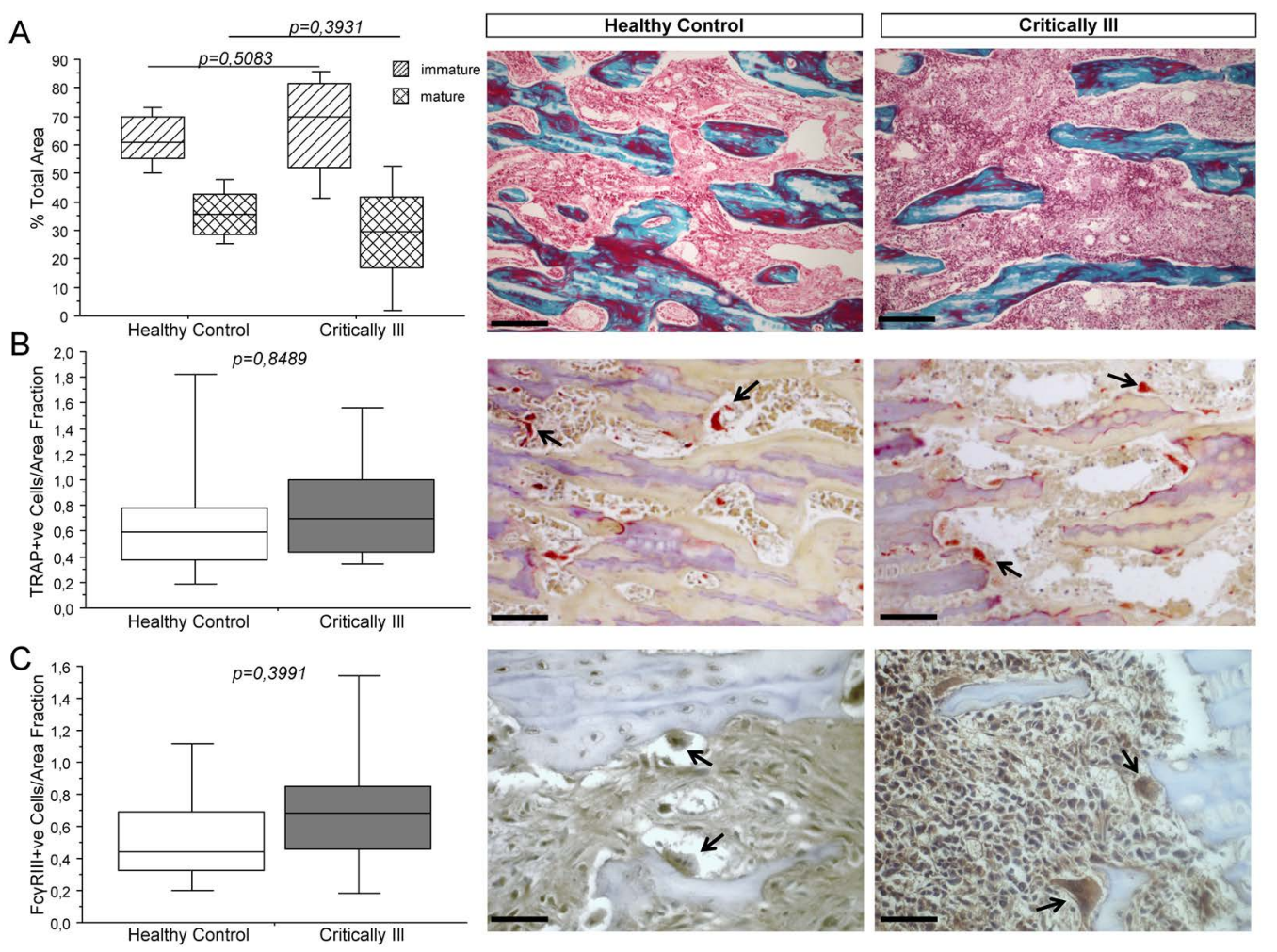


A

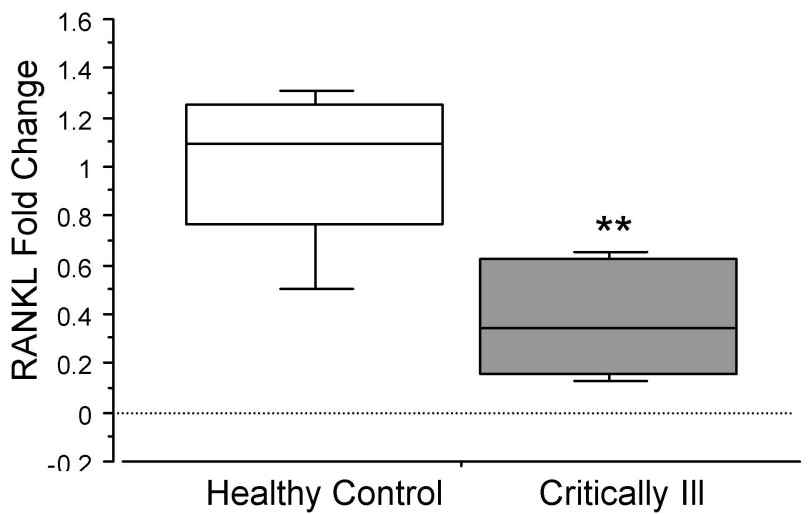

D

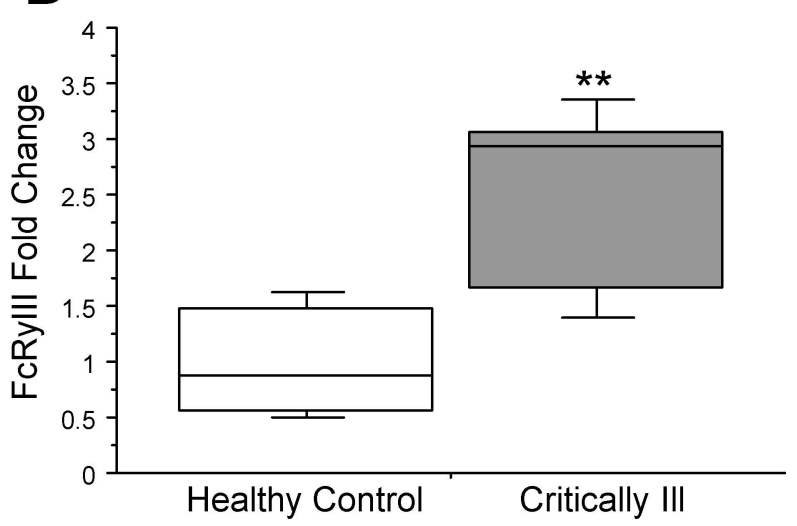

B

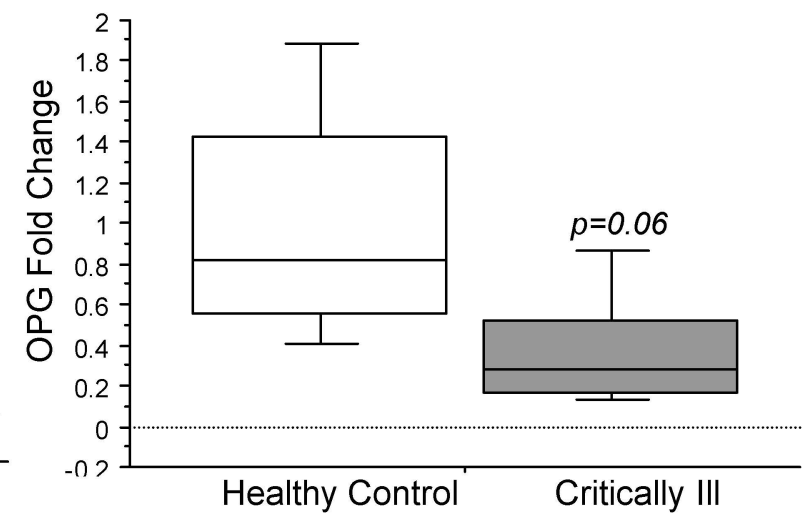

E

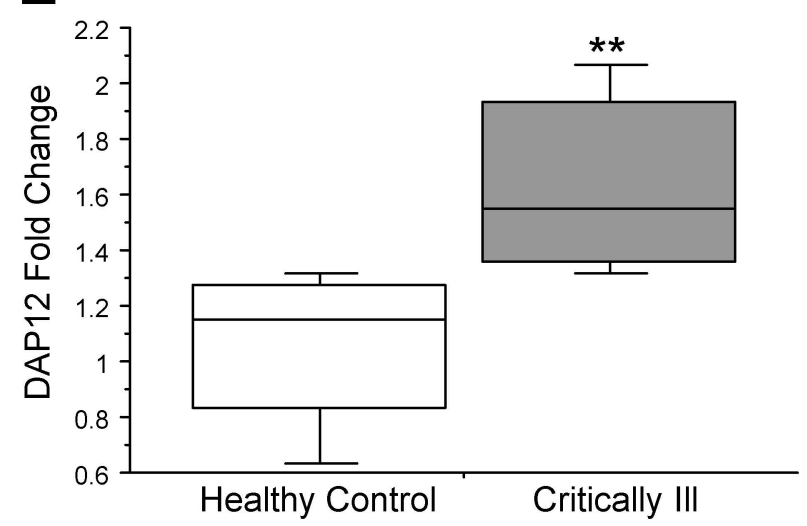

C

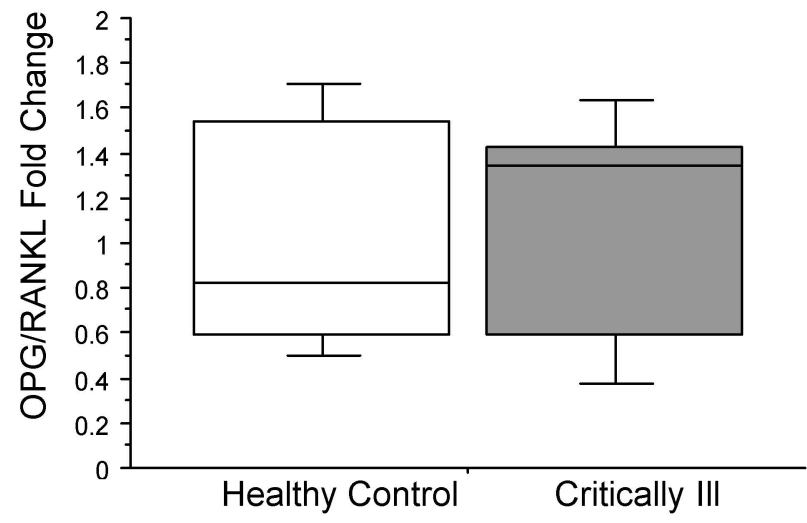




\section{Osteoblast}

VEGFR1( $\downarrow$ )

VEGFR2 $\downarrow$
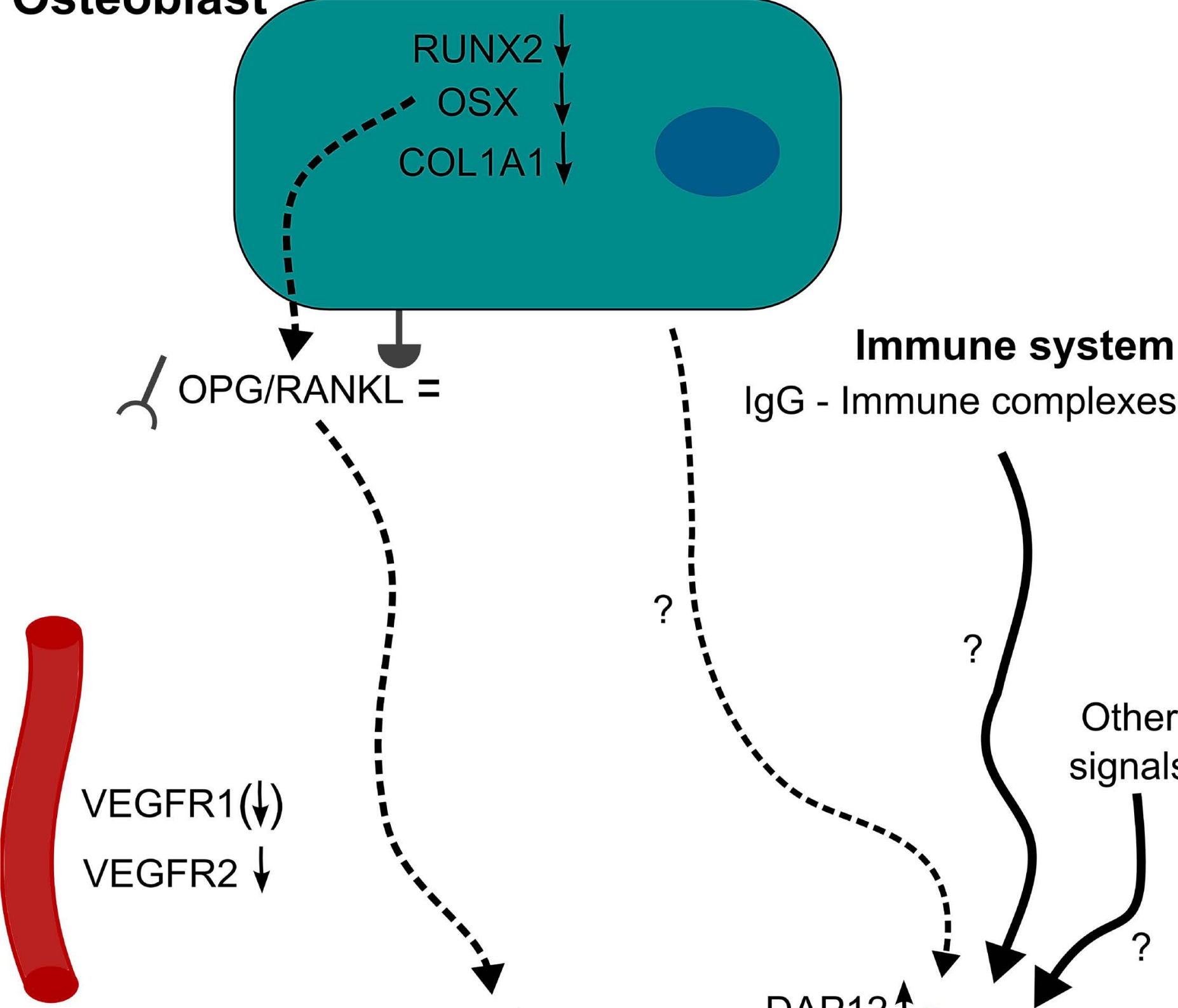

IgG - Immune complexes

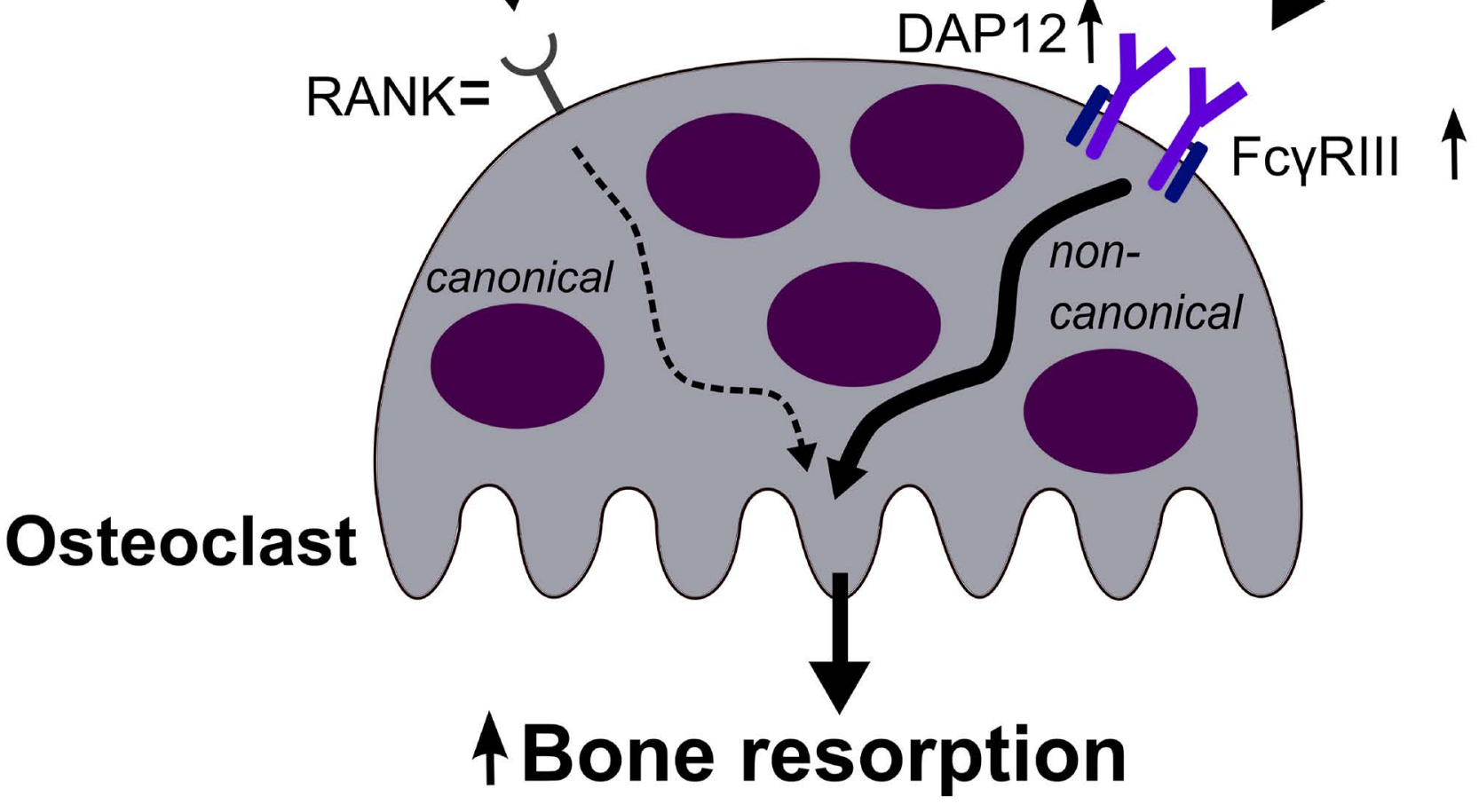


Table S1. Sequences of qPCR primers.

\begin{tabular}{|c|c|}
\hline GENE NAME & SEQUENCE \\
\hline \multicolumn{2}{|l|}{ Osteogenic markers } \\
\hline \multirow{2}{*}{ RUNX2 } & Forward: 5'- CTTCACAAATCCTCCССAAG-3' \\
\hline & Reverse: 5'- ATGCGCCCTAAATCACTGAG-3' \\
\hline \multirow{2}{*}{ OSX } & Forward: 5’- ATGGCGTCCTCTCTGCTTG-3' \\
\hline & Reverse: 5’- GCTTCTTCGTGCCTGCTTT-3’ \\
\hline \multirow{2}{*}{ COL1A1 } & Commercial gene expression assay, Applied Biosystems \\
\hline & Reference number: OC03396116_A1 \\
\hline \multicolumn{2}{|l|}{ Osteoclastogenic markers } \\
\hline \multicolumn{2}{|c|}{ Canonical RANK/RANKL/OPG pathway } \\
\hline \multirow{2}{*}{ OPG } & Forward: 5’-GTGCACTTGGTTTGCTGCTA-3' \\
\hline & Reverse: 5‘-CCTGAAGAATGCCTCCTCAC-3’ \\
\hline \multirow{2}{*}{ RANK } & Forward: 5’- GCAGTTCCTCTCTGCCATCT-3’ \\
\hline & Reverse: 5’- AGTGCCTTCCCTTGTTTCCT-3’ \\
\hline \multirow{2}{*}{ RANKL } & Forward: 5'-AAAATGAAGATTTGCAAGACG-3' \\
\hline & Reverse: 5’-TTTCTCGGCTCTCAGGTGTT-3’' \\
\hline \multicolumn{2}{|c|}{ Non-canonical ITAM signaling pathway } \\
\hline \multirow{2}{*}{ DAP12 } & Forward: 5’- CTGGTGTTGACCCTGCTCAT-3' \\
\hline & Reverse: 5’-TGACTCGGTCTCAGTGATGC-3' \\
\hline \multirow{2}{*}{ Fc $\gamma$ RIII } & Forward: 5'- GACCTCCACATTCCAGAAGC-3' \\
\hline & Reverse: 5‘-GAGAACGGAGGACGAGATGA-3’ \\
\hline \multicolumn{2}{|l|}{ Angiogenic markers } \\
\hline \multirow{2}{*}{ VEGFR1 } & Forward: 5'-CAAGCCGGACAGACACTGTA-3' \\
\hline & Reverse: 5'-GCAGGTGGATTTCGAGATGT-3' \\
\hline \multirow{2}{*}{ VEGFR2 } & Forward: 5'-GCAAGAAGGGTTTCAGCATC-3' \\
\hline & Reverse: 5'-GCTCACCAGCACGTCATAAA-3' \\
\hline \multicolumn{2}{|l|}{ Housekeeping gene } \\
\hline \multirow{2}{*}{ HPRT } & Forward: 5'-TGTAGATTTTATCAGACTGAAGAGCTACTGT-3' \\
\hline & Reverse: 5’- AAGGAAAGCAAGGTCTGCATTGTT-3’ \\
\hline
\end{tabular}

